\title{
Caracterização tecnológica das zeólitas naturais associadas às rochas eruptivas da Formação Serra Geral, na região de Piraju-Ourinhos (SP)
}

\author{
Mirian Chieko Shinzato ${ }^{1}$, Tarcísio José Montanheiro², Valdecir de Assis Janasi ${ }^{3}$, \\ Francisco de Assis Negri' ${ }^{2}$, Jorge Kazuo Yamamoto \& Sandra Andrade $^{3}$
}

\begin{abstract}
Resumo Ocorrências de zeólitas associadas às rochas eruptivas da Formação Serra Geral são encontradas na região de Piraju-Ourinhos (SP). Considerando a importância das propriedades tecnológicas desses minerais, como na retenção de contaminantes em solos e águas, foram caracterizados dois tipos de amostras: o dacito (ZD) e as zeólitas que preenchem suas amígdalas (ZP). Foram determinadas a composição química e mineralógica, a superfície específica, a micromorfologia e a capacidade de troca catiônica (CTC). A caracterização mineralógica de ZP revelou presença de mordenita, e na amostra de dacito amigdaloidal (ZD): mordenita, quartzo, sanidina e plagioclásio sódico. A fórmula química obtida para a mordenita foi: $\mathrm{Na}_{0,33} \mathrm{~K}_{1,4} \mathrm{Ca}_{0,82} \mathrm{Ba}_{0,12}$ $\mathrm{Sr}_{0,03}\left[\mathrm{Al}_{4} \mathrm{Si}_{20,1} \mathrm{O}_{48}\right] .11,38 \mathrm{H}_{2} \mathrm{O}$, e a relação $\mathrm{Si} / \mathrm{Al}$ igual a 5,02 - valor típico de mordenitas de simetria ortorrômbica do tipo $\mathrm{Cmcm}$. Valores de superfícies específicas revelaram que a área interna - onde grande parte da troca iônica ocorre - corresponde a 93,70\% da superfície total em ZP e 89,83\% em ZD. A imagem da mordenita em microscópio eletrônico de varredura revelou agregados de fibras alongadas e claras. Valores de CTC determinados para a mordenita e o dacito amigdaloidal foram, respectivamente, iguais a 1,2 e 0,1 meq/g. Comparando a CTC teórica da mordenita (obtida pela fórmula química) que é de 2,2 meq $/ \mathrm{g}$, com os valores experimentais, observa-se que a sua troca iônica foi incompleta, provavelmente pelo fato do cátion trocador $\left(\mathrm{K}^{+}\right)$ocupar canais que dificultam seu deslocamento. Verifica-se, portanto, que o arranjo estrutural da zeólita controla e limita sua propriedade de troca catiônica.
\end{abstract}

Palavras-chave: zeólita, dacito, caracterização tecnológica, capacidade de troca catiônica.

\begin{abstract}
Technological characterization of the natural zeolites associated with eruptive rocks from the Serra Geral Formation, Paraná Sedimentary Basin, Piraju-Ourinhos region. Zeolites associated with eruptive rocks from the Serra Geral Formation occur in the Piraju-Ourinhos region (SW of State of São Paulo). These zeolites were selected for a study of their technological properties in retaining some contaminants in soil and water. Two samples were analysed: the amygdaloidal dacite (ZD) and a concentrate of zeolites (ZP) extracted from the amygdales in this rock. The chemical and mineralogical composition, the surface area, the micromorphology and the cationic exchange capacity (CEC) of these samples were determined. The mineralogical analyses show that the zeolite concentrate (ZP) corresponds mostly to mordenite; the other main minerals present in ZD are quartz, sanidine and sodic plagioclase. The chemical formula determined for the mordenite is: $\mathrm{Na}_{0.33}$ $\mathrm{K}_{1.4} \mathrm{Ca}_{0,82} \mathrm{Ba}_{0.06} \mathrm{Sr}_{0.015}\left[\mathrm{Al}_{4.01} \mathrm{Si}_{20.11} \mathrm{O}_{48}\right] .11 .41 \mathrm{H}_{2} \mathrm{O}$; the $\mathrm{Si} / \mathrm{Al}$ ratio of 5.02 indicates an orthorrombic symmetry and space group $\mathrm{Cmcm}$. Surface area determinations indicate that the internal areas of the samples- where most of the ion exchange reactions occur - correspond to $93.7 \%$ (ZP) and $89.8 \%$ (ZD) of their total specific surface area. Scanning electron microscopy reveal that the mordenite is formed by aggregates of light-colored long fibers. The CEC are, respectively, $1.2 \mathrm{meq} / \mathrm{g}(\mathrm{ZP})$ and $0.1 \mathrm{meq} / \mathrm{g} \mathrm{ZD}$ ), while the theoretical CEC of the mordenite (obtained from its chemical formula) is $2.2 \mathrm{meq} / \mathrm{g}$. This indicates that the ionic exchange capacity of the studied mordenite is not complete, which is probably related to the position of the exchanger cation $\left(\mathrm{K}^{+}\right)$, occupying channels in the mineral structure, which controls and limits its mobility.
\end{abstract}

Keywords: zeolite, dacite, technological characterization, cationic exchange capacity.

INTRODUÇÃO Nos últimos 50 anos, a família das zeólitas tem sido alvo de estudos de identificação mineral e caracterização tecnológica, tendo em vista as excelentes qualidades que as suas propriedades físico-químicas oferecem para o uso e aplicação em diversas atividades humanas. Em particular, ressalta-se a utilização de ze- ólitas naturais no tratamento de efluentes industriais e domiciliares e, na recomposição de solos contaminados por metais pesados, devido às suas propriedades de adsorção e de troca iônica (Kalló 2001; Beyazit et al.2003; Pitcher et al. 2004; Jimenez et al. 2004; Wingenfelder et al. 2005). Acrescente-se a isso a sua aplicação como car-

1 - Departamento de Ciências Exatas e da Terra, Univ. Federal de São Paulo, Diadema (SP), Brasil. Email: mirianshinzato@hotmail.com 2 - Instituto Geológico da Secretaria do Meio Ambiente do Estado de São Paulo, São Paulo (SP), Brasil. Email: tjmonta@igeologico.sp.gov.br; negri@igeologico.sp.gov.br

3 - Instituto de Geociências, Univ. de São Paulo, São Paulo (SP), Brasil. Email: vajanasi@usp.br.; jkyamamo@usp.br; sandrade@usp.br 
ga na indústria do papel e, na indústria cimenteira, como cimento pozolânico ou material diretamente aplicado no concreto (Duarte et al. 2002).

Neste trabalho, são apresentados os resultados de uma pesquisa direcionada à identificação e caracterização das zeólitas naturais associadas às rochas eruptivas da Formação Serra Geral, que afloram na região de Piraju-Ourinhos (SP) ou, mais especificamente, no município de Timburi-SP.

Contexto geológico A ocorrência de zeólita de Timburi/SP (Fig. 1) está associada a dacitos amigdaloidais que pertencem à Formação Serra Geral da Bacia Sedimentar do Paraná. Eles ocupam, aproximadamente, 65 x $20 \mathrm{Km}$ de uma área alongada na direção N40W que se estende entre o Rio Paranapanema (altitude local de 450 até $550 \mathrm{~m}$ ) e a Serra da Fartura, com elevações acima de 800 m (Janasi et al. 2007).

Os dacitos são as rochas eruptivas mais setentrionais da Bacia do Paraná e constituem, localmente, a base da pilha vulcânica, em contato direto com sedimentos eólicos da Formação Botucatu. O dacito, em direção aos contatos superior e inferior, tem caráter vítreo, e é tipicamente mais rico em vesículas, as quais são preenchidas apenas parcialmente por zeólitas fibrosas, calcita e variedades de sílica microcristalina. Quando em contato com o arenito, a matriz vítrea passa do cinza-escuro ao marrom-"chocolate". O litotipo assim formado corres- ponde a uma rocha de coloração freqüentemente marrom acastanhado porfirítica com fenocristais milimétricos de plagioclásio, e matriz vítrea fortemente vesicular (Fig. 2). As vesículas existentes facilmente ultrapassam $1 \mathrm{~cm}$, com possibilidade de atingir mais de $5 \mathrm{~cm}$ de diâmetro, e variam quanto ao achatamento e preenchimento, que geralmente é de zeólitas, quartzo (localmente ametista),

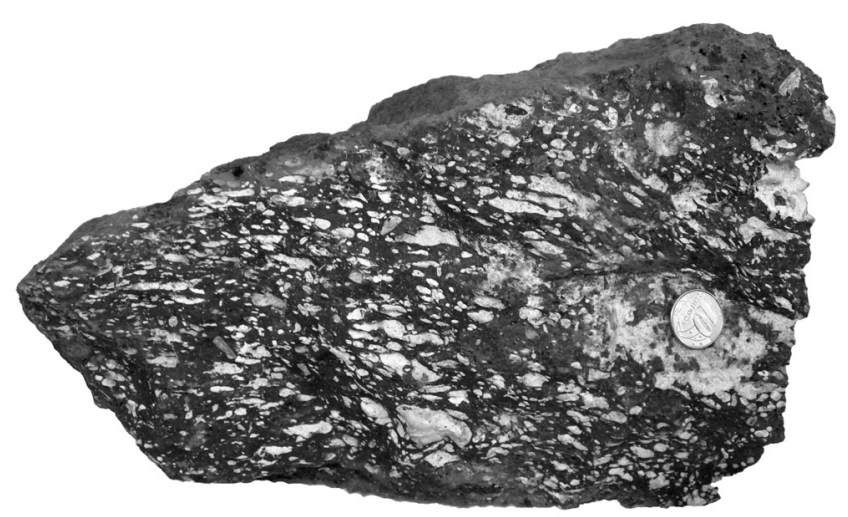

Figura 2 - Foto do dacito amigdaloidal portador de zeólita da Formação Serra Geral (Bacia Sedimentar do Paraná).

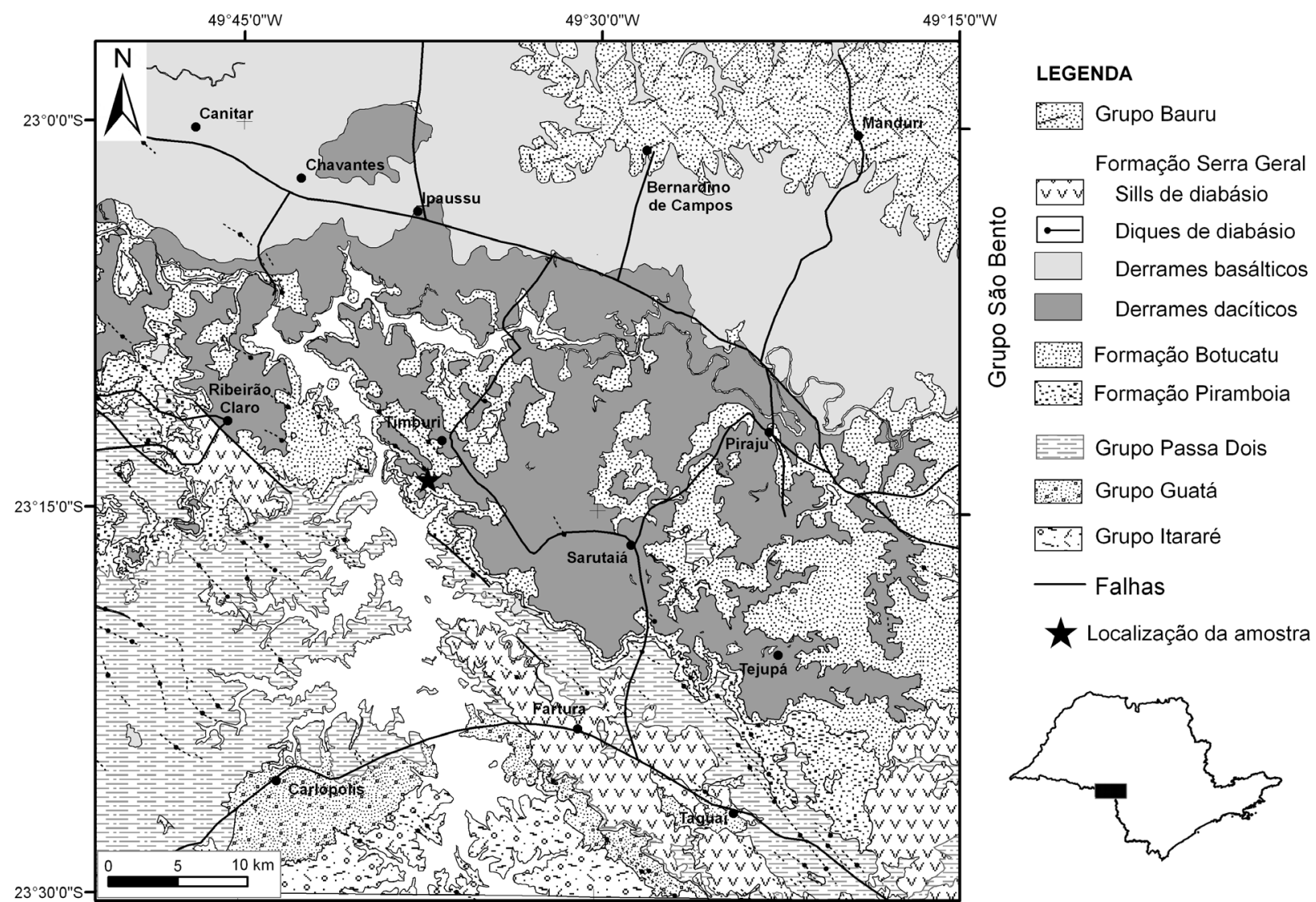

Figura 1 - Localização e mapa geológico da região de Timburi (SP) (fonte: Negri et al. 2006). 
calcedônea ou localmente calcita.

Zeólitas: aspectos gerais Zeólitas são aluminossilicatos hidratados formados por estruturas tridimensionais de tetraedros de $\mathrm{SiO}_{4}$ e de $\mathrm{AlO}_{4}$, cuja deficiência de carga - gerada pela substituição isomórfica do $\mathrm{Si}^{4+}$ pelo $\mathrm{Al}^{3+}$ - é compensada por cátions alcalinos e/ou alcalinos terrosos situados nas cavidades dos anéis de oxigênio. Nessas cavidades, a água e outras moléculas podem-se alojar e/ou movimentar, permitindo hidratação reversível e uma troca iônica equivalente à substituição do $\mathrm{Si}^{4+}$ por $\mathrm{Al}^{3+}$ nos tetraedros estruturais (Pabalan \& Bertetti 2001).

A uniformidade das cavidades formadas pelos anéis de oxigênio nas zeólitas contrasta com a relativa variedade de tamanho observada nos poros de uma sílica gel, alumina ativada e carvão ativado, permitindo-lhe a capacidade de troca catiônica (CTC) de 2 a 4 $\mathrm{meq} / \mathrm{g}$, ou seja, aproximadamente duas vezes a CTC de uma argila bentonita (Mumpton 1999). Assim sendo, ao contrário da maioria dos trocadores iônicos não-cristalinos (resinas orgânicas e gels inorgânicos aluminossilicáticos), a zeólita impõe uma seletividade entre os íons competidores.

A estrutura microporosa das zeólitas faz com que elas apresentem uma superfície interna muito grande em relação à externa, e lhes permite a transferência de massa entre o espaço cristalino e o meio externo, mas que pode ser limitada pelo diâmetro dos poros da estrutura zeolítica (Aguiar et al. 2002).

Mumpton (1984) observou que o processo de adsorção do metal é influenciado pela natureza da zeólita, sendo, por conseguinte, fundamental que se conheça a sua composição química, CTC, tamanho dos poros e outras características mais específicas. Outra particularidade que pode levar à diminuição da CTC da zeólita e, até, tornar impossível a troca, ocorre quando os cátions ficam presos em algumas partes estruturais, como nas cavas sodalitas, por exemplo.

De modo geral, as zeólitas com baixa relação $\mathrm{Si} / \mathrm{Al}$ têm as maiores capacidades de troca, apesar de algumas exceções, devido à presença de impurezas ou variações na composição (Breck 1984). Colella (1996) verificou que zeólitas com baixa relação $\mathrm{Si} / \mathrm{Al}$ possuem carga estrutural relativamente elevada e preferência por cátions de maior valência e de raio iônico pequeno, pelo fato de existir distância média menor entre os sítios aniônicos $\left(\mathrm{AlO}_{2}\right)$ adjacentes da estrutura zeolítica (Bosco et al. 2004).

A distribuição geológica dos minerais de zeólita é restrita e está associada, preferencialmente, às rochas de origem eruptiva ou sedimentar e, raramente formam depósitos minerais economicamente explotáveis como aqueles conhecidos em Cuba, China, Japão e outros poucos países.

No cenário nacional, Rezende \& Angélica (1997) destacam três ocorrências: zeólitas na Formação Corda (Bacia do Parnaíba, MA/TO); analcima na Formação Adamantina (Bacia do Paraná, SP) e heulandita na Formação Botucatu (Bacia do Paraná, MS). Além dessas, os autores ainda mencionam as ocorrências da
Formação Uberaba na Bacia do Paraná e aquelas da Formação Macau na Bacia Potiguar. Destacam-se, ainda, as zeólitas associadas aos basaltos amigdaloidais da Formação Serra Geral (Bacia do Paraná) descritas por Franco (1952) e Murata et al. (1987), entre outros.

O Brasil ainda não registra a lavra deste bem mineral, apesar do reconhecimento de um prospecto com cerca de $4 \times 10^{9} \mathrm{t}$ de minério associado a arenitos da Formação Corda na Bacia do Parnaíba, Estado do Maranhão (Rezende 2002). A zona zeolítica da Formação Corda compreende um pacote de arenitos eólicos e fluviais onde duas espécies de zeólitas - estilbita e laumontita - são os constituintes mais abundantes do cimento, que corresponde à 20 - 40\% da rocha (Rezende 2002).

MATERIAIS E MÉTODOS Os estudos das propriedades tecnológicas foram executados em frações de duas amostras volumétricas coletadas em afloramentos típicos da Formação Serra Geral, na área do município de Timburi (SP): a primeira, amostra ZP (zeólita pura), é constituída por zeólitas extraídas diretamente das cavidades dos dacitos com auxílio de espátula de metal; a segunda, amostra ZD (dacito amigdaloidal), é constituída pelo dacito amigdaloidal, rocha hospedeira das zeólitas. As duas amostras (ZP e ZD) foram pulverizadas até a granulometria inferior a $200 \mathrm{mesh}$, respectivamente, em moinhos de ágata e em disco de fricção. Seguiram-se os ensaios para caracterização tecnológica que incluíram: determinação da composição química e mineralógica, determinação da superfície específica (SE), análise micromorfológica por meio de microscopia de varredura (MEV) e determinação da capacidade de troca catiônica (CTC).

CARACTERIZAÇÃO TECNOLÓGICA A associação mineralógica foi identificada mediante técnicas de difração de raios X, cujos difratogramas foram gerados por um equipamento Siemens D500 do Laboratório de Difração de raios X do Instituto de Geociências da Universidade de São Paulo (IGc-USP). A composição química das amostras foi obtida por fluorescência de raios X no aparelho Philips/PW2400 do Laboratório de Caracterização Tecnológica do Departamento de Engenharia de Minas e Petróleo da Escola Politécnica da USP.

A determinação da superfície específica (SE) das amostras pulverizadas se processou segundo duas técnicas de análise. A primeira, conhecida como método de adsorção de $\mathrm{N}_{2}$ (BET) - realizada no Laboratório de matérias-primas particuladas e sólidos não metálicos do Departamento de Engenharia Metalúrgica e de Materiais da Escola Politécnica da USP - determina somente a superfície da área externa do mineral (Inel \& Tümsek 2000; Ruthven 2001). A segunda técnica - proposta por Bower \& Gschwend (1952) - permite medir as áreas externa e interna do mineral por saturação com etilenoglicol. Nesse procedimento, considerou-se que cada 1 mg de etileno-glicol é capaz de recobrir uma área de 3,22 $\mathrm{m}^{2}$, conforme Grohmann (1972).

Para verificar a feição morfológica da zeólita 
estudada, frações da amostra ZP foram observadas em microscópio eletrônico de varredura em um aparelho LEO/440, equipado com espectrômetro de energia dispersiva ( $\mathrm{Si}-\mathrm{Li}$ ) da Oxford do Laboratório de Microscopia Eletrônica do IGc-USP.

Nos ensaios para determinação da capacidade de troca catiônica (CTC) utilizou-se a técnica em que as amostras são previamente saturadas com solução de acetato de sódio $(\mathrm{N})$ e, em seguida, com solução de acetato de amônio (N). Primeiramente, com a finalidade de deslocar os íons originalmente presentes nas cavidades das zeólitas pelo $\mathrm{Na}^{+}$, foram preparadas misturas de 0,4 g de cada amostra e $40 \mathrm{~mL}$ de solução de acetato de sódio $(\mathrm{N})$ que foram mantidas sob agitação durante 2 horas, à temperatura ambiente e, em seguida, centrifugadas e filtradas. Na segunda etapa, as amostras saturadas com sódio foram misturadas com $40 \mathrm{~mL}$ de solução de acetato de amônio $(\mathrm{N})$ e agitadas por 2 horas, à temperatura ambiente, para provocar o deslocamento do $\mathrm{Na}^{+}$ pelo $\mathrm{NH}_{4}^{+}$. As soluções coletadas após a centrifugação e filtragem foram encaminhadas para análise via ICPOES (ARL/3410) no Laboratório de Química e ICP do IGc-USP. Os valores de $\mathrm{Na}^{+}$quantificados nas soluções representam os teores dos íons retidos na estrutura da zeólita que, posteriormente, foram deslocados pelo amônio. Os teores expressos na unidade miligrama por litro $(\mathrm{mg} / \mathrm{L})$ foram convertidos em miliequivalente por grama (meq/g) para expressarem os respectivos valores da capacidade de troca catiônica.

\section{RESULTADOS E DISCUSSÃO}

Composição mineralógica e química Os estudos de caracterização mineralógica da amostra ZP identificaram como principal componente a zeólita mordenita, ao passo que na amostra de dacito amigdaloidal (ZD) a composição é de mordenita, quartzo, sanidina e plagioclásio sódico.

A mordenita é uma das oito zeólitas naturais consideradas suficientemente abundantes para a exploração comercial que, no entanto, há várias décadas vem sendo substituída por suas formas sintéticas (Mortier et al. 1978). De acordo com Armbruster \& Gunter (2001), a estrutura cristalina da mordenita assemelha-se a folhas franzidas, cujos anéis são formados por seis tetraedros paralelos a (100) (Fig. 3a). As folhas estão ligadas por quatro tetraedros que permitem a definição de dois

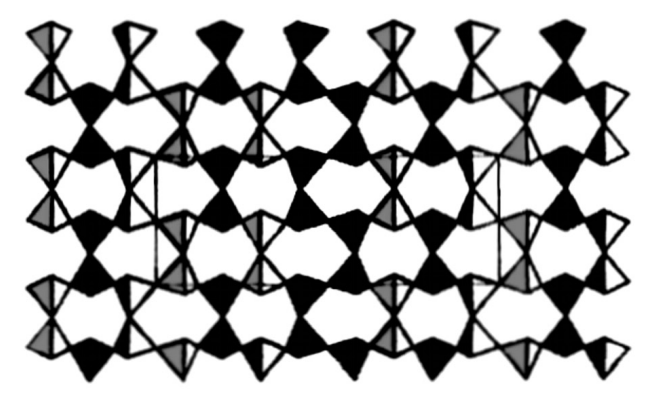

a
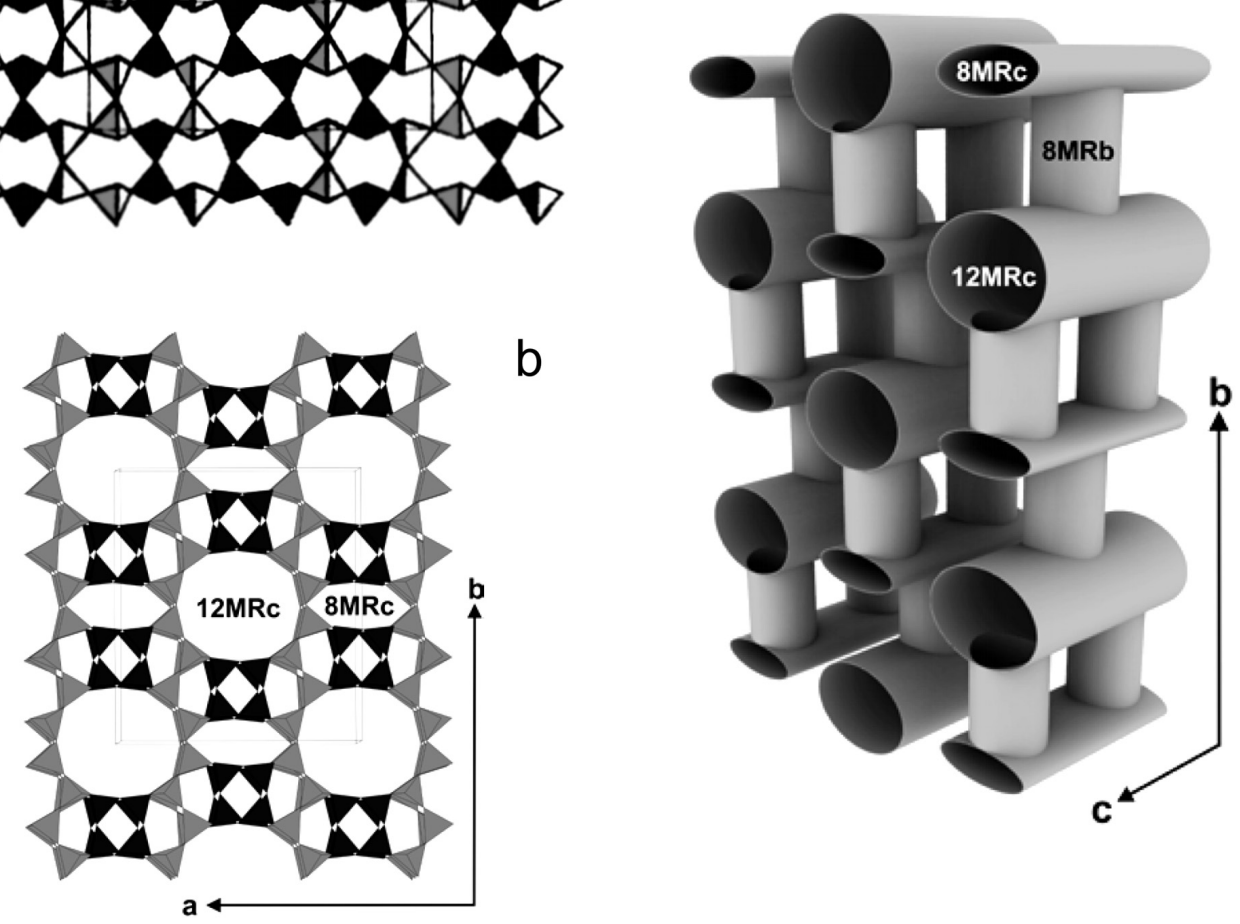

C

Figura 3 - Estrutura da mordenita: (a) folhas pararelas à (100): metade dos ápices dos tetraedros aponta para cima; a outra metade, para baixo (fonte: Armbruster \& Gunte 2001); (b) folhas conectadas ao longo do eixo b constituidas por 4 tetraedros (cinza escuro), formando anéis com 12 tetraedros (12MRc) e 8 tetraedros (8MRc) - poros comprimidos - ao longo do eixo c (fonte: Simoncic \& Armbruste 2004); (c) sistema de canais na mordenita onde se pode observar suas conexões, e a estrutura oferecendo passagem apenas em uma única direção, para a difusão dos grandes íons ou moléculas (fonte: Simoncic \& Armbruste 2004). 
tipos de séries de canais: (1) paralelos ao eixo c (Fig. $3 \mathrm{~b}$ ), de formato elipsoidal com abertura de $6,5 \times 7,0$ $\AA$, constituído por 12 tetraedros (12MRc) e, ainda de 8 tetraedros (8MRc), fortemente comprimidos, com abertura de 2,6 x 5,7 Å; e (2) paralelos ao eixo b (Fig. 3c), formado por 8 tetraedros $(8 \mathrm{MRb})$ de abertura de $3,4 \mathrm{x}$ $4,8 \AA$, que conecta os grandes canais com aqueles fortemente comprimidos (Simoncic \& Armbruster 2004).

Esse tipo de sistema com diferentes poros encontrado na mordenita - constituído por canais largos ligados por canais mais estreitos - favorece a difusão bidimensional de pequenas moléculas como $\mathrm{N}_{2}, \mathrm{O}_{2}$, etc., mas restringe a difusão unidimensional de moléculas maiores, como as orgânicas (Braga \& Morgon 2007).

Nas mordenitas, os cátions compensadores de carga ocupam três sítios principais: dois estão nos pequenos canais conectores, paralelos ao eixo b (8MRb), próximos dos anéis formados pelos 4 tetraedros e, o terceiro, no centro do canal formado por 12 tetraedros (12MRc) (Armbruster \& Gunter 2001).

Apesar das mordenitas apresentarem, efetivamente, canais largos (12MRc da Fig. 3), na maioria das formas naturais, estes se tornam pequenos, seja devido à localização dos cátions compensadores de carga ou ainda, à presença de material amorfo (impurezas) no interior dos poros e, ainda, às falhas no empilhamento estrutural (Simoncic \& Armbruster 2004). Segundo os mesmos autores, tais falhas provocam o rompimento da continuidade dos grandes canais e, dessa forma, permitem somente a entrada de moléculas com diâmetro menor que 4,5 $\AA$ em suas cavidades.

Uma característica comum na composição química da zeólita é a presença de $\mathrm{O}, \mathrm{Si}, \mathrm{Al}, \mathrm{Ca}, \mathrm{Mg}, \mathrm{Ba}$, $\mathrm{Na}, \mathrm{K}$ e $\mathrm{H}$ como elementos fundamentais, e de $\mathrm{Fe}, \mathrm{Sr}$, Li, $\mathrm{Be}, \mathrm{Cs}, \mathrm{Cu}$ e $\mathrm{Pb}$ como elementos subordinados ou ocasionais (Passaglia \& Sheppard 2001). Para esses autores, a quantidade de ferro nas zeólitas é desprezível por ser proveniente de impurezas, não devendo ser considerada no cálculo de sua fórmula química. A ausência desse elemento evidencia-se também na coloração das zeólitas que são predominantemente brancas ou pálidas.

A fórmula geral da zeólita pode ser representada como $M_{x} D_{y}\left[A l_{x+2 y} S i_{n-(x+2 y)} \mathrm{O}_{2 n}\right] \cdot \mathrm{mH}_{2} \mathrm{O}$, onde $\mathrm{M}$ corresponde aos cátions monovalentes e $D$ aos bivalentes; os elementos encontrados no interior dos colchetes ocupam a estrutura tetraédrica e os da parte externa representam os cátions trocáveis, que neutralizam a carga negativa da estrutura; as moléculas de água presentes são, por fim, as que geralmente coordenam os cátions trocáveis (Gottardi \& Galli 1985).

Com o resultado da análise química da amostra ZP, calculou-se a fórmula química da mordenita, tomando por base 48 átomos de oxigênio (Tab. 1), de acordo com o método proposto por Jackson et al. (1967); não foram incluídos nesse cálculo os óxidos de teor inferior a $0,1 \mathrm{mg} / \mathrm{L}$, por serem considerados impurezas.

Assim, de acordo com os valores apresentados na tabela 1 , a fórmula química da mordenita está representada a seguir:
Tabela 1 - Composição química (\% peso) da mordenita (amostra ZP) e os respectivos ions de sua fórmula à base de 48 átomos de oxigênio.

\begin{tabular}{c|c|c|c}
\hline óxidos & $\%$ & Íon & íon/fórmula \\
\hline $\mathrm{SiO}_{2}$ & 67,85 & $\mathrm{Si}^{4+}$ & 20,081 \\
$\mathrm{Al}_{2} \mathrm{O}_{3}$ & 11,48 & $\mathrm{Al}^{3+}$ & 4,003 \\
$\mathrm{CaO}$ & 2,59 & $\mathrm{Ca}^{2+}$ & 0,821 \\
$\mathrm{Na}_{2} \mathrm{O}$ & 0,58 & $\mathrm{Na}^{+}$ & 0,332 \\
$\mathrm{~K}_{2} \mathrm{O}$ & 3,70 & $\mathrm{~K}^{+}$ & 1,398 \\
$\mathrm{BaO}$ & 1,03 & $\mathrm{Ba}^{2+}$ & 0,12 \\
$\mathrm{SrO}$ & 0,17 & $\mathrm{Sr}^{2+}$ & 0,03 \\
& & $\mathrm{O}^{2-}$ & 48,00 \\
$\mathrm{H}_{2} \mathrm{O}^{+}$ & 12,60 & $\mathrm{H}_{2} \mathrm{O}$ & 11,38 \\
\hline
\end{tabular}

$\mathrm{Na}_{0,33} \mathrm{~K}_{1,4} \mathrm{Ca}_{0,82} \mathrm{Ba}_{0,12} \mathrm{Sr}_{0,03}\left[\mathrm{Al}_{4} \mathrm{Si}_{20,1} \mathrm{O}_{48}\right] .11,38 \mathrm{H}_{2} \mathrm{O}$

Para garantir a validade da composição mineral da zeólita, calculou-se o valor do erro (E\%), segundo a equação (1) (Gottardi \& Galli 1985).

$$
\mathrm{E}(\%)=\left[\mathrm{Al}-\mathrm{Al}_{\text {térico }}\right] /\left[\mathrm{Al}_{\text {teórico }}\right]
$$

onde,

[Al] = ion/fórmula do alumínio multiplicado pela sua valência.

[ $\left.\mathrm{Al}_{\text {térico }}\right]=$ soma dos valores de ion/fórmula dos cátions monovalentes e bivalentes multiplicados pelas respectivas cargas.

De acordo com Gottardi \& Galli (1985) valores de $\mathrm{E}(\%)$ superiores a $10 \%$ invalidam a fórmula. Considerando a composição química proposta para a mordenita estudada, verificou-se que o seu E (\%) é de 2,27\%, validando-na.

$\mathrm{O}$ valor da relação $\mathrm{Si} /(\mathrm{Si}+\mathrm{Al})$ das mordenitas (razão $R$ ) pode variar entre 0,81 e 0,86 nas amostras amigdaloidais e entre 0,80 e 0,85 nas sedimentares (Passaglia \& Sheppard 2001). A amostra estudada apresenta $R$ igual a 0,83 , ou seja, dentro da faixa mencionada pelos autores acima para os dois tipos de origens.

A análise da composição química da mordenita permite verificar que a relação $\mathrm{Si} / \mathrm{Al}$ é de 5,02 e confirma os valores propostos por Colella (1996) para este tipo de zeólita, que estariam entre 4,19 e 5,79. Ainda, segundo os estudos de Braga \& Morgon (2007), as mordenitas com $\mathrm{Si} / \mathrm{Al}$ por volta de 5 possuem simetria ortorrômbica do tipo $\mathrm{Cmcm}$.

Segundo a fórmula da mordenita estudada, o $\mathrm{K}^{+}$é o principal cátion compensador de cargas, seguido do $\mathrm{Ca}^{2+}$, $\mathrm{Na}^{+}, \mathrm{Ba}^{2+} \mathrm{e} \mathrm{Sr}^{2+}$. Segundo Mortier et al. (1978), o K trocável de zeólitas com estrutura do tipo da mordenita (grupo espacial $\mathrm{Cmcm}$ ) ocupam, comumente, os canais interconectores (8MRb) (Fig. 3c). Ressalta-se que nessa posição, 
o cátion trocável apresenta dificuldades de deslocamento durante as reações de troca com os íons do meio externo, tornando esse processo possivelmente incompleto.

Superfície específica (SE) e análise morfológica A superfície específica externa e total (externa e interna) das amostras obtidas, respectivamente, pelas técnicas de adsorção de $\mathrm{N}_{2}$ (BET) e de etileno-glicol permitiram a determinação da superfície interna (Tab. 2).

Os dados da tabela 2 evidenciam que a superfície específica total da amostra ZP é maior, por ser ela constituída apenas por mordenita - o que justifica o baixo valor obtido na amostra ZD (dacito amigdaloidal), composta de 10 a $20 \%$ de mordenita.

Observa-se que a área interna nas três amostras analisadas, corresponde a $93,70 \%$ da superfície total na zeólita pura (ZP) e 89,83\% no dacito amigdaloidal (ZD). Assim sendo, espera-se que a propriedade de adsorção (diretamente relacionada à superfície específica) seja maior no interior dos poros, e principalmente em ZP.

A análise da imagem de mordenitas, obtida por meio do microscópio eletrônico de varredura (MEV), revelou que a morfologia da zeólita estudada é formada por agregado de fibras longas e claras (Fig. 4).

Capacidade de troca catiônica (CTC) Os resultados da capacidade de troca catiônica das amostras de mordenita e das rochas portadoras de zeólitas são apresentados na tabela 3. Pabalan \& Bertetti (2001) mostraram que a capacidade de troca catiônica teórica pode ser obtida com a fórmula química da zeólita mediante a determinação do número de equivalente do(s) cátion(s) trocável(is) ou, simplesmente, a partir do peso molecular do $\mathrm{Al}^{1+}$ presente na amostra (Tab. 3).

As amostras de dacito amigdaloidal (ZD) apresentou $8,3 \%$ da capacidade de troca catiônica obtida na zeólita pura (ZP). Esse baixo valor era esperado, uma vez que, além de zeólitas (10 a 20\% no dacito amigdaloidal), a amostra é constituída principalmente por outros minerais como quartzo e feldspatos, que não contribuem para o aumento da propriedade de troca iônica.

$\mathrm{O}$ valor de CTC, obtido por meio de saturação com acetato de sódio seguido de acetato de amônio, corresponde a $54,5 \%$ daquele determinado a partir do conteúdo de $\mathrm{Al}^{3+}$ da fórmula química da mordenita $(\mathrm{ZP})$, ou seja, 2,2 meq/g. Esses dados corroboram os obtidos por Townsend \& Loizidou (1984), que pesquisaram o equilíbrio de troca entre $\mathrm{Na}^{+}$e $\mathrm{NH}_{4}^{+}$de mordenitas naturais de Lovelock (Nevada) e os compararam com a CTC calculada a partir do conteúdo de $\mathrm{Al}^{3+}(2,11 \mathrm{meq} / \mathrm{g})$. Os autores observaram uma eficiência de $50,1 \%$ e concluíram que, nas mordenitas naturais, a troca iônica entre $\mathrm{Na}^{+} \mathrm{e}$ $\mathrm{NH}_{4}^{+}$ocorre de forma reversível, porém incompleta. Por outro lado, verifica-se que, apesar das mordenitas sintéticas (AW-300 e Zeolon) analisadas por Ames (1964) serem as mais puras, elas apresentaram capacidade de troca catiônica igual, respectivamente, a 1,6 e 1,9 meq/g, não atingindo o valor teórico de $2,11 \mathrm{meq} / \mathrm{g}$.

Considerando que os cátions trocáveis das zeólitas podem ocupar sítios cristalográficos distintos e,
Tabela 2 - Superficies especificas (SE) externa e total das amostras $Z P$ e $Z D$ obtidas, respectivamente, pelos métodos de adsorção de $N_{2}$ (BET) e de etileno-glicol, e de superficie especifica interna (calculada pela diferença dos valores de BET e etileno-glicol). Comparativamente, são citados alguns valores de SE de mordenitas naturais determinados por Hernández et al. (2000) (a) e Covarrubias et al. (2006) (b), a partir das isotermas de adsorção de $N_{2}$ do método de BET.

\begin{tabular}{|c|c|c|c|c|}
\hline \multicolumn{4}{|c|}{ Amostras analisadas } & \multirow{2}{*}{$\begin{array}{l}\text { Valores de referência de } \\
\qquad \begin{array}{c}\text { SE da mordenita } \\
\text { natural }\left(\mathrm{m}^{2} / \mathrm{g}\right)\end{array}\end{array}$} \\
\hline Método & $\begin{array}{c}\mathrm{SE} \\
\left(\mathrm{m}^{2} / \mathrm{g}\right)\end{array}$ & $\mathrm{ZP}$ & ZD & \\
\hline BET & externa & 12,37 & 6,41 & $120,5^{(\mathrm{a})} ; 277^{(\mathrm{b})}$ \\
\hline $\begin{array}{l}\text { Etilenoglicol } \\
\text { - BET }\end{array}$ & interna & 183,93 & 56,6 & \\
\hline Etilenoglicol & total & 196,30 & 63,01 & \\
\hline
\end{tabular}

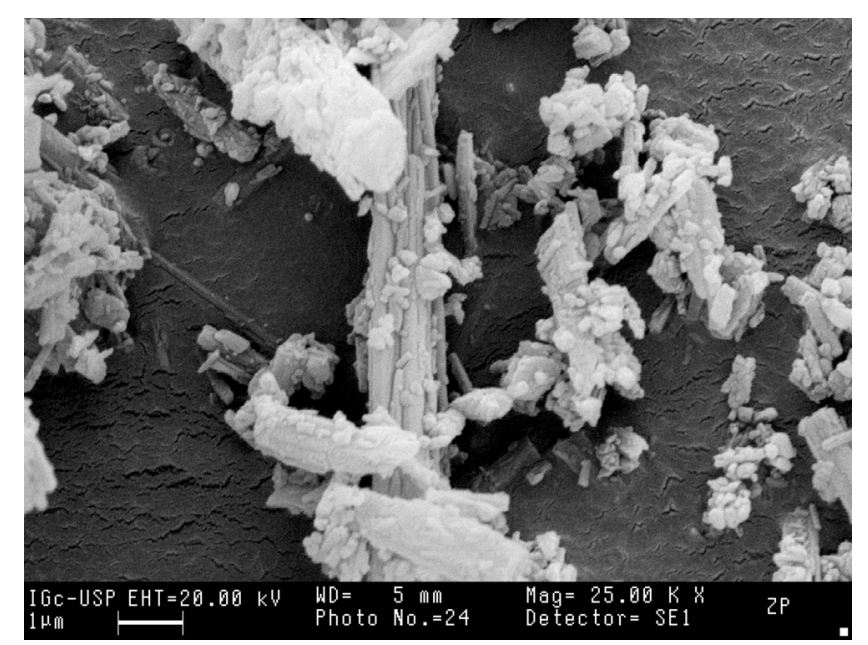

Figura 4 - Fotomicrografia obtida por meio de MEV da mordenita (amostra ZP).

Tabela 3 - Valores de capacidade de troca cationica obtidos experimentalmente para as amostras $Z P$ e $Z D$ segundo o método de saturação com acetato de $\mathrm{Na}^{+} /$ $\mathrm{NH}_{4}^{+}$. A CTC de mordenitas sintéticas AW-300 e Zeolon foram retirados de Ames (1964) para comparação. A CTC teórica foi calculada a partir do número de moles do alumínio obtido na fórmula química proposta para a mordenita.

\begin{tabular}{c|c|c}
\hline Amostras & $\begin{array}{c}\text { CTC experimental } \\
(\mathrm{meq} / \mathrm{g})\end{array}$ & $\begin{array}{c}\text { CTC teórica } \\
(\mathrm{meq} / \mathrm{g})\end{array}$ \\
\hline ZP & 1,2 & 2,2 \\
ZD & 0,1 & - \\
AW-300 & 1,6 & 2,11 \\
Zeolon & 1,9 & 2,11 \\
\hline
\end{tabular}


conseqüentemente, apresentar diferentes propriedades de seletividade iônica, a reação incompleta de troca iônica na amostra analisada pode ter ocorrido em virtude de o íon $\mathrm{K}^{+}$(principal cátion compensador de cargas) ocupar os canais $8 \mathrm{MRb}$ (Fig. 3) e dificultar, devido ao seu arranjo estrutural, o acesso dos cátions trocadores. Cabe mencionar, ainda, que algumas características comuns presentes nas zeólitas naturais como, presença de impurezas e defeitos na estrutura cristalina, também podem interferir na eficiência da capacidade de troca iônica do mineral (Simoncic \& Armbruster 2004).

Apesar da propriedade de troca iônica não ter sido completa, a CTC obtida para a mordenita é semelhante às CTCs dos argilominerais expansivos como vermiculita e esmectitas que, de acordo com Souza Santos (1975), gira em torno de $1,0 \mathrm{meq} / \mathrm{g}$, ao passo que a CTC do dacito corresponde a da caulinita, igual a $0,1 \mathrm{meq} / \mathrm{g}$.

CONCLUSÕES As zeólitas naturais associadas aos dacitos amigdaloidais da região de Timburi (SP) foram identificadas como do tipo mordenita, rica em $\mathrm{K}$ e, com elevada relação $\mathrm{Si} / \mathrm{Al}(5,02)$. A sua composição química e relação Si/Al estão condizentes com exemplares de outras partes do mundo.

Em conseqüência do sistema poroso da mordenita e, principalmente pelo fato do $\mathrm{K}^{+}$ocupar os canais $8 \mathrm{MR}$, de difícil acesso, a propriedade de capacidade de troca pelos cátions $\mathrm{Na}^{+}$e $\mathrm{NH}_{4}^{+}$pode ter sido afetada.
Razão esta que explicaria o motivo pelo qual se obteve, na prática, valor de CTC em torno de $1,2 \mathrm{meq} / \mathrm{g}$, inferior à CTC teórica igual a $2,2 \mathrm{meq} / \mathrm{g}$. Esse valor, no entanto, ainda é elevado e corresponde às CTCs de algumas argilas expansivas como vermiculita e esmectitas.

Por outro lado, a rocha total apresenta em torno de $8,3 \%$ da CTC da mordenita, o que corresponde ao valor de $0,1 \mathrm{meq} / \mathrm{g}$ (semelhante à argila caulinita).

A superfície específica determinada para a mordenita estudada $\left(196 \mathrm{~m}^{2} / \mathrm{g}\right)$ ficou na média dos valores encontrados na literatura - 120,5 $\mathrm{m}^{2} / \mathrm{g}$ segundo Hernández et al. (2000) e $277 \mathrm{~m}^{2} / \mathrm{g}$ de acordo com Covarrubias et al. (2006) -; e a área interna revelou ser maior que a externa, indicando que nessa parte do mineral (área interna) ocorre a maioria das reações de troca.

Por fim, verificou-se que apesar da literatura pertinente indicar valores elevados de CTC para as zeólitas em geral - baseando-se na sua fórmula química nem sempre, na prática, esse valor é alcançado, devido a vários fatores, como natureza e localização do cátion compensador de cargas, defeitos estruturais e presença de impurezas; revelando-se assim a importância da caracterização tecnológica desses minerais.

Agradecimentos Os autores agradecem à Fapesp pelo financiamento da pesquisa (processo 03/06259-4).

\section{Referências}

Aguiar M.R.M.P., Novaes A.C., Guarino A.W.S. 2002. Remoção de metais pesados de efluentes industriais por aluminossilicatos. Quim. Nova, 25(6B):1145-1154.

Ames L.L. Jr. 1964. Some zeolite equilibria with alkali metal cations. Am. Mineral., 49:127-145.

Armbruster T., Gunter M.E. 2001. Crystal structures of natural zeolites. In: Bish D.L. \& Ming D.W. (eds.) Reviews in Mineralogy and Geochemistry. Mineralogical Society of America, 45:1-67.

Beyazit N., Peker I., Ergun O.N. 2003. Removal of lead and zinc from aqueous solution using Amasya zeolites from Turkey. International Journal of Environment and Pollution, 19(2): 160-170.

Bosco S.M.D., Jimenez R.S., Carvalho W.A. 2004. Aplicação da zeólita natural escolecita na remoção de metais pesados de efluentes industriais: competição entre os cátions e processo de dessorção. Ecl. Quím., 29(1):47-56.

Bower C.A. \& Gschwend F.B. 1952. Ethylene glycol retention by soils as a measure of surface area and interlayer swelling. Soil Science Society of America Journal, 16:342-345.

Braga A.A.C. \& Morgon N.H. 2007. Descrições estruturais cristalinas de zeólitos. Quím. Nova, 30(1):178-188.

Breck D.W. 1984. Zeolite Molecular Sieve. New York, John Wiley \& Sons, $771 \mathrm{p}$.

Colella C.1996. Ion exchange equilibria in zeolite minerals. Mineral. Deposita, 31:554-562.

Covarrubias C., Garcia R., Arriagada R., Yánez J., Garland
M.T. 2006. Cr (III) exchange on zeolites obtained from kaolin and natural mordenite. Micropor. Mesopor. Mater., 88:220-231.

Duarte A.C.P., Monte M.B.M., Luz A.B., Leal G.P. 2002. Aplicação de zeólita natural como adsorvente de metais pesados presentes em efluentes industriais. In: CETEM, Encontro Nacional de Tratamento de Minérios e Metalurgia Extrativa em Recife (PE), 19, Atas de Comunicações técnicas, 8p. Disponivel em: www.cetem.gov.br/ publicacao/CTs/CT2002-072-00.pdf

Franco R.R. 1952. Zeólitas dos basaltos do Brasil Meridional (Gênese e Paragênese). Boletim da USP.: Fac. Fil. Cienc. Letras, 150(Mineralogia 10):5-69.

Gottardi G. \& Galli E. 1985. Natural Zeolites. Berlin, Springer-Verlag, 409p.

Grohmann F. 1972. Superfície específica. In: Moniz A.C. (ed.) Elementos em pedologia. São Paulo, editora Polígono, p.111-122.

Hernández M.A., Corona L., Rojas F. 2000. Adsorption characteristics of natural erionite, clinoptilolite and mordenite zeolites from Mexico. Adsorption, 6:33-45.

Inel O. \& Tümsek E. 2000. The measurement of surface areas of some silicates by solution adsorption. Turk $J$. Chem., 24:9-19.

Jackson E.D., Stevens R.E., Bowen R.W. 1967. A computer-based procedure for deriving mineral formulas from mineral analyses. Geological Survey Professional Paper, 575-C:23-31. 
Janasi V.A., Negri F.A., Montanheiro T.J., Freitas V.A., Rocha B.C., Reis P.M. 2007. Geochemistry of the eocretacic basalt magmatism in the Piraju-Ourinhos region, SE Brazil, and implications to the stratigraphy of the Serra Geral Formation. Rev. Bras. Geoc., 37:148-162.

Jimenez R.S., Bosco S.M.D., Carvalho W.A. 2004. Remoção de metais pesados de efluentes aquosos pela zeólita natural escolecita - influência da temperatura e do $\mathrm{pH}$ na adsorção em sistemas monoelementares. Quím. Nova, 27(5):734-738.

Kalló D. 2001. Applications of natural zeolites in water and wastewater treatment. In: Bish D.L. \& Ming D.W. (eds.) Reviews in Mineralogy and Geochemistry. Mineralogical Society of America, 45:519-550.

Mortier W.M., Pluth J.J., Smith J.V. 1978. Positions of cations and molecules in zeolites with the mordenit-type framework. IV. Dehydrated and rehydrated K-exchanged "ptilolite". In: Sand L.B., Mumpton F.A. (eds.) Natural Zeolites: occurremce, properties, use. New York, Pergamon Press, p.53-62.

Mumpton F.A.1984. The rol of natural Zeolites in agriculture and aquaculture. In: Pond W.G. \& Mumpton F.A. (eds.) ZeoAgriculture. Use of natural Zeolites in agriculture and aquaculture. Bulder, CO, USA, Westview Press, p. 3-27.

Mumpton F.A.1999. La roca magica: uses of natural zeolites in agriculture and industry. Proc. Natl. Acad. Sci. USA, 96:3463-3470.

Murata K.J., Formoso M.L.L., Roisenberg A. 1987. Distribution of zeolites in lavas of Southeastern Parana Basin, State of Rio Grande do Sul, Brazil. J. Geology, 95:455467.

Negri F.A., Montanheiro T.J., Janasi V.A., Reis P.M. 2006. Mapa de distribuição das rochas vulcãnicas nas folhas Piraju/SP e Jacarezinho/SP-PR. In: SBG, Congr. Bras. Geol., 43, Aracaju, Anais, p.247.

Pabalan R.T., Bertetti F.P. 2001. Cation-exchange properties of natural zeolites. In: Bish D.L. \& Ming D.W. (eds.)
Reviews in Mineralogy and Geochemistry. Mineralogical Society of America, 45:453-518.

Passaglia E. \& Sheppard R.A. 2001. The crystal chemistry of zeolites. In: Bish D.L. \& Ming D.W. (eds.) Reviews in Mineralogy and Geochemistry. Mineralogical Society of America, 45:69-116.

Pitcher S.K., Slade R.C.T., Ward N.I. 2004. Heavy metal removal from motorway stormwater using zeolites. Sci. Total Environ., 334-335:161-166.

Rezende N.G.A.M. 2002. A zona zeolítica da Formação Corda - Bacia do Parnaíba. Dissertação de Mestrado, Universidade Federal do Pará, 141p.

Rezende N.G.A.M. \& Angélica R.S. 1997. Geologia das zeolitas sedimentares no Brasil. In: Schobbenhaus C., Queiroz E. T., Coelho C.E.S. (eds.) Principais Depósitos Minerais do Brasil. Brasília, DNPM/CPRM, v.4-B. p.193-212.

Simoncic P. \& Armbruster T. 2004. Peculiarity and defect structure of the natural and sybthetic zeolite mordenite: a single-crystal study. Amer. Mineral., 89:421-431.

Souza Santos P. 1975. Tecnologia de Argilas. São Paulo, Edgard Blücher Ltda, 1:340p.

Ruthven D.M. 2001. Characterization of zeolites by sorption capacity measurements. In: Robson H. (ed.) Verified syntheses of zeolitic materials. $2^{\text {nd }}$ revised edition, Elsevier Science B.V., p.61-65.

Townsend R.P. \& Loizidou M. 1984. Ion-exchange properties of natural clinoptilolite, ferrierite and mordenite. I. Sodium-ammonium equilibria. Zeolites, 4:191-195.

Wingenfelder U., Hansen C., Furrer G., Schulin R. 2005. Removal of heavy metals from waters by natural zeolites. Environ. Sci. Technol., 39(12):4606-4613.

Manuscrito ID 10881

Submetido em 13 de março de 2008 Aceito em 20 de setembro de 2008 Sistema eletrônico de submissão 